\title{
Scalar-relativistic effects in solids in the framework of a Douglas-Kroll transformed Dirac-Coulomb Hamiltonian
}

\author{
Norbert J.M. Geipel, Bernd A. Heß \\ Institut für Physikalische und Theoretische Chemie, \\ Wegelerstr. 12, Universität Bonn, D-53115 Bonn, Germany
}

(Received 11 April 1997)

\begin{abstract}
The Douglas-Kroll transformed Dirac-Coulomb Hamiltonian is used to describe scalar-relativistic effects in solids. A Hartree-Fock approximation with periodic boundary conditions makes it feasible to use methods originally developed for atoms and molecules to solve the corresponding equations for crystalline systems. The implementation is realized within the CRYSTAL program. Scalar-relativistic effects in silver compounds of FCC structure such as $\mathrm{AgF}, \mathrm{AgCl}, \mathrm{AgBr}$ and $\mathrm{Ag}$ are investigated for the molar formation energy, the lattice parameter, the isothermal bulk modulus and the pressure derivative.
\end{abstract}

Typeset using REVTEX 


\section{INTRODUCTION}

In the last decade, methods for treating relativistic effects in heavy atoms and molecules have been developed in order to extend the applicability of ab initio quantum chemical methods to heavy atoms and molecules. Recently, different approaches and their realizations were compared as concerning the accuracy and efficiency of the method [1]. A detailed discussion of the accuracy of different relativistic approximations by the investigation of hydrogenic energy levels can be found in Ref. [2]. The Douglas-Kroll (DK) transformed Dirac-Coulomb Hamiltonian [3,4] is considered to be an efficient and reliable approximation for calculations on relativistic systems. The theoretical framework of this approximation is based on quantum electrodynamics, and as one main consequence this relativistic Hamiltonian is variationally stable and therefore has normalizable bound-state solutions. The application of external-field projection operators which project onto the positive-energy states of the Dirac single-particle Hamiltonian leads to a two-component formalism which is separable in a spin-free and a spin-dependent part. There has been an extensive discussion of relativistic effects in the framework of this approach for atoms and molecules such as silver and gold [5.6], gold hydride [6,7] and gold fluoride [8] using the Hartree-Fock (HF) approximation as well as various methods including electron correlation effects and spin-orbit effects [9].

There is also a plenitude of methods for treating relativistic effects in solids. The mainstream approach in this field being density functional theory (DFT) in the local density approximation (LDA). All these methods are, in principle, based on a relativistic extension of the Hohenberg-Kohn theorem [10]. Most of these approaches rely on the four-component treatment of a spherical symmetric potential (see Refs. [11,12] and references therein), which can be relatively easily solved using appropriate methods from atomic theory, extended to effective one-particle equations given by MacDonald and Vosko [13]. One possibility to elude the shape approximation for the crystal potential are relativistic effective-core potentials (ECP) which are adjusted to (quasi-) relativistic atomic calculations (see Ref. [14] and references therein) and have to be transferable to the corresponding crystalline situation 
15, 16, 17.

The extension of HF methods in solid-state theory to the relativistic case [18 is less obvious, however in practice, the treatment of potentials of non-spherical symmetry (and the non-local exchange potential) requires an effort which is much larger than in the nonrelativistic case. Consequently, it seems to be a reasonable idea to use the DK transformation for the one-electron operators also in HF calculations with periodic boundary conditions. In this Letter we describe a corresponding implementation of the method in the CRYSTAL88 program [19,20] and report first applications of the code.

\section{METHOD}

The spin-free part of the DK Hamiltonian is used to treat scalar-relativistic effects in solids. Using atomic units (with the electron mass $m=1$ ), the relativistic operator has the following form:

$$
\begin{aligned}
H_{+}^{\mathrm{sf}_{1}} & =\sum_{i}\left[E_{i}-m c^{2}+V_{\mathrm{eff}}^{\mathrm{sf}}(i)\right]+\sum_{i<j} \frac{1}{r_{i j}}, \\
E_{i} & =\left(p_{i}^{2} c^{2}+m^{2} c^{4}\right)^{\frac{1}{2}}, \\
V_{\mathrm{eff}}^{\mathrm{sf}}(i) & =-A_{i}\left[V_{\mathrm{ext}}(i)+\mathbf{R}_{i} V_{\mathrm{ext}}(i) \mathbf{R}_{i}\right] A_{i}-\frac{1}{2}\left\{\left\{E_{i}, W_{1}^{\mathrm{sf}}(i)\right\}, W_{1}^{\mathrm{sf}}(i)\right\}, \\
\text { with } A_{i} & =\sqrt{\frac{m c^{2}+E_{i}}{2 E_{i}}}, \mathbf{R}_{i}=\frac{c \mathbf{p}_{i}}{m c^{2}+E_{i}} .
\end{aligned}
$$

The brackets $\{$,$\} denote the anticommutator \{a, b\}=a b+b a$. The operator $W_{1}^{\text {sf }}$ is an integral operator with kernel

$$
\mathcal{W}_{1}^{\mathrm{sf}}\left(\mathbf{p}_{i}, \mathbf{p}_{i}^{\prime}\right)=A_{i}\left(\mathbf{R}_{i}-\mathbf{R}_{i}^{\prime}\right) A_{i}^{\prime} \frac{V_{\mathrm{ext}}\left(\mathbf{p}_{i}, \mathbf{p}_{i}^{\prime}\right)}{E_{i}+E_{i}^{\prime}}
$$

Within this approach the use of the non-relativistic Coulomb repulsion instead of the transformed two-electron term is a good approximation if scalar-relativistic effects are considered 司. 
An all-electron ab initio HF approximation for crystalline systems has been realized in the CRYSTAL88 program [19,20]. To construct a Fock operator in an atomic orbital (AO) basis (direct space representation) within this approach including the above relativistic operators, three main steps are necessary:

1. In order to represent the operator $\mathbf{R}_{i} V_{\text {ext }}(i) \mathbf{R}_{i}$ in Eq. (酉) in an AO basis, we have to calculate matrix elements of the operator $\mathbf{p}_{i} V_{\text {ext }}(i) \mathbf{p}_{i}$.

2. The Bloch function $(\mathrm{BF})$ representation of the kinetic energy, the external potential and the additional matrix elements mentioned above is used to get a representation of the relativistic operators in the $\mathrm{BF}$ basis.

3. To get a representation of the relativistic operators in direct space, those quantities are Fourier transformed back to the AO basis.

We make use of the fact that scalar-relativistic effects are short-range [16. Therefore, the long-range tail of the electrostatic potential calculated in the CRYSTAL88 program by means of Ewald sums [21] is not relativistically corrected. Rather, the relativistic contribution of the external potential described by Eq. (四) to the Fock operator is restricted to a finite number of direct lattice vectors $\mathbf{h}$ within the spatial region that is treated by the direct lattice series of the Ewald potential function [21] - hereafter, dubbed "direct space potential zone".

Each AO is expanded in real spherical Gaussian-type functions (SGTF) 20,22]. We denote by $[\omega \mathbf{g}]=\chi_{\Omega}\left(\mathbf{r}-\mathbf{g}-s_{\omega}\right)$ the $\omega$ th $\mathrm{AO}$ of the primitive unit cell identified by the vector $\mathrm{g}$ located on an atom whose image in the primitive unit cell is assigned by the fractional vector $s_{\omega} ; \mathbf{g}, \mathbf{h}, \ldots$ label direct lattice vectors and $\Omega=(n, l, m)$ denotes the quantum numbers characterizing the AO. For simplicity we write 1,2 for $\omega_{1}, \omega_{2}$ in the following. To evaluate the integrals, we calculate additional matrix elements of $V_{\mathrm{ext}}^{C}(\mathbf{r})=\sum_{\mathbf{h}}^{C} \sum_{a} Z_{a}\left|\mathbf{r}-\mathbf{h}-\mathbf{s}_{a}\right|^{-1}$ in a basis of SGTF of the form

$$
R(12 \mathbf{g})=<\nabla 1 \mathbf{O}\left|V_{e x t}^{C}(\mathbf{r})\right| \nabla 2 \mathbf{g}>
$$

These integrals result from the operator $\mathbf{R}$, which is equal to the linear momentum $\mathbf{p}=-i \nabla$ 
times a $p^{2}$-dependent kinematical factor. In practice, we calculate these derivatives with Cartesian Gaussian-type functions (CGTF) and transform the corresponding integrals to SGTF afterwards 22].

In order to adapt the basis set to the translational invariance of the crystal, the AOs are used to build BFs [20,23]. It is convenient to use this basis to get a matrix representation of non-local operators such as $E, A$ and $\left(E+m c^{2}\right)^{-1}$ because by definition this is the basis in which the matrix of one-electron operators is symmetry blocked. When the BF representation of the kinetic energy operator $\widehat{T}$

$$
\widehat{T}(12 \mathbf{k})=S(12 \mathbf{k})^{-1 / 2} T(12 \mathbf{k}) S(12 \mathbf{k})^{-1 / 2}
$$

is expressed in its eigenbasis

$$
\widehat{T}(12 \mathbf{k})=U(12 \mathbf{k}) \lambda(12 \mathbf{k}) U^{\dagger}(12 \mathbf{k})
$$

— the diagonal matrix $\lambda(12 \mathbf{k})$ contains the positive real eigenvalues of $\widehat{T}(12 \mathbf{k})$ and the unitary matrix $U(12 \mathbf{k})$ the corresponding eigenvectors - the BF representation of an analytic function $f(\widehat{T}(12 \mathbf{k}))$ corresponding to operators like $\widehat{E}(12 \mathbf{k}), \widehat{A}(12 \mathbf{k})$ and $\left(E \widehat{+} m c^{2}\right)^{-1}(12 \mathbf{k})$ is given by

$$
f(\widehat{T}(12 \mathbf{k}))=U(12 \mathbf{k}) f(\lambda(12 \mathbf{k})) U^{\dagger}(12 \mathbf{k}) .
$$

Here we assume that this relationship holds approximately also in the finite matrix representation given by the AO basis set, from which the BFs are constructed. The contribution of those operators to the relativistic external potential $V_{\text {eff }}^{C \text {,sf }}(12 \mathbf{k})$ given by Eq. (1) — restricted to the direct space potential zone — is then realized by simple matrix multiplication for every $\mathbf{k}$ point within the irreducible wedge of the first Brillouin zone (IBZ).

The relativistic external potential $V_{\mathrm{eff}}^{C, \mathrm{sf}}(12 \mathbf{k})$ transforms like a totally symmetric operator. To transform this operator back to the AO basis, the Fourier transformation of $V_{\text {eff }}^{C, \text { sf }}(12 \mathbf{k})$ restricted to the IBZ (see Refs. [20,23] and references therein) generates a matrix

$$
\Pi_{\text {eff }}^{C, \mathrm{sf}}(12 \mathbf{g})=\int_{I B Z} V_{\mathrm{eff}}^{C, \mathrm{sf}}(12 \mathbf{k}) \exp (i \mathbf{k} \cdot \mathbf{g}) d \mathbf{k}
$$


from which the full potential matrix $V_{\mathrm{eff}}^{C \text { sf }}(12 \mathrm{~g})$ is generated by applying the appropriate rotation matrices [20,23]. The relativistic operator $E(12 \mathbf{k})$ is treated likewise.

The Fock operator expressed in an AO basis can formally be written as sum of a oneelectron and a two-electron part

$$
F(12 \mathbf{g})=H(12 \mathbf{g})+B(12 \mathbf{g})
$$

The contribution of Eq. (四) to the Fock operator is related to $H(12 \mathbf{g})$ and does not include any relativistic correction of the long-range behaviour of the electrostatic potential $Z(12 \mathrm{~g})$ because it is restricted to the direct space potential zone. We thus include relativistic effects due to the external potential by adding the difference of the relativistic potential and the non-relativistic potential calculated in this zone to the total non-relativistic potential as calculated in the CRYSTAL88 program.

$$
H(12 \mathbf{g})=E(12 \mathbf{g})+V_{\text {eff }}^{C, \mathrm{sf}}(12 \mathbf{g})-V_{\mathrm{ext}}^{C}(12 \mathbf{g})+Z(12 \mathbf{g}) .
$$

Thus, the delicate treatment of the Coulomb series [21] remains the same. As usual the total HF energy per primitive unit cell is given by

$$
\epsilon=\frac{1}{2} \sum_{1,2, \mathbf{g}} P(12 \mathbf{g})(2 H(12 \mathbf{g})+B(12 \mathbf{g}))
$$

where $P(12 \mathbf{g})$ denotes the density matrix of a crystal [24].

\section{TECHNICAL DETAILS}

Scalar-relativistic effects in several simple silver compounds of FCC structure such as $\mathrm{AgF}, \mathrm{AgCl}, \mathrm{AgBr}$ and $\mathrm{Ag}$ are investigated using this method with the reoptimized uncontracted basis sets for $\mathrm{F}, \mathrm{Cl}$ and $\mathrm{Br}$ of Dunning [25] and for $\mathrm{Ag}$ of Martin [26]. In crystalline compounds low-exponent functions are likely to introduce linear dependencies. Therefore, diffuse exponents $(\leq 0.04)$ of the basis set for $\mathrm{Ag}$ are discarded. Concerning the question whether HF solutions for conductors are appropriate in spite of the vanishing density of 
states at the Fermi level and the related problem of finding appropriate HF solutions of the free electron gas, we refer to Ref. [24] and references therein. Basis sets and details of the atomic and the CRYSTAL88 HF calculations using $H_{+}^{\mathrm{sf}_{1}}$ in the relativistic case and resulting total HF energies are given in Tables \and ㅍ.

All calculations are realized close to program limitations and whereever possible parameters for high-precision calculations 20] are used. To realize an accurate representation of the relativistic operators, it is convenient to use relatively large uncontracted basis sets. In order to obtain a reliable transformation from indirect space (k space) to direct space, the number of $\mathbf{k}$ points used to realize the integration over the IBZ should also be large. Testing the method described above for $T(12 \mathbf{k})$ and $V_{\text {ext }}^{C}(12 \mathbf{k})$ by comparing its IBZ integration with the given integrals of $T(12 \mathbf{g})$ and $V_{\text {ext }}^{C}(12 \mathbf{g})$, it turned out that $29 \mathbf{k}$ points of the Monkhorst net [20] within the IBZ are sufficient to obtain an absolute error for the integrals which is less than $10^{-8}$. Moreover, the change in the total HF energy per primitive unit cell for metallic silver is less than $0.9 \times 10^{-4}$ au if we use $72 \mathrm{k}$ points instead of $29 \mathrm{k}$ points within the IBZ for the relativistic version of the program.

The geometry optimization is realized with the restart option GEOM of the CRYSTAL88 program [20]. Two different equations of state (EOS) deduced by Murnaghan and Birch, respectively, 27 are used to fit the total energy as a function of the lattice parameter. Though nearly all the results are only weakly dependent on the applied EOS - except the pressure derivatives of $\mathrm{Ag}$ and $\mathrm{AgBr}$ - the accuracy of the fit and especially related results such as bulk moduli and pressure derivatives have to be investigated carefully (for details see Table [II]).

A corresponding ECP calculation of $\mathrm{AgCl}$ [29] is repeated (see Table [V]) to ensure compatibility of all used parameters. Since small-core pseudopotentials for Ag are used, it is assumed that frozen-core effects are small and relativistic all-electron HF calculations are comparable with corresponding ECP calculations. 


\section{RESULTS}

Although relativistic effects are widely discussed in literature (see Ref. [28] and references therein), ab initio calculations of silver halides with FCC structure including scalarrelativistic corrections are rare [30] and in most cases pseudopotential approaches are used to consider those effects (see Ref. [29] and references therein). By way of contrast, due to the simple structure of diamagnetic metals relativistic calculations of metallic silver are numerous (see Refs. [16,17] and references therein). Nevertheless, scalar-relativistic effects without any substantial approximation of the crystal potential have not been investigated before.

The results of the investigated FCC crystals are collected in Table एII. Properties such as the lattice parameters and the related molar volumes show small effects - they decrease up to $2.0 \%$ respectively up to $5.8 \%$ (for $\mathrm{Ag}$ ) as compared to the non-relativistic values. The corresponding decrease of the nearest-neighbor distances lies between $0.8 \mathrm{pm}$ for $\mathrm{AgF}$ and $6.4 \mathrm{pm}$ for $\mathrm{Ag}$ - relativistic bond-length contractions of similar molecules are larger [28.31]. Looking at the molar HF binding energies, a remarkable bond destabilization of the halides is observed - the molar HF binding energies are higher up to 8.9\% (for $\mathrm{AgF}$ ) as compared to the non-relativistic values. This trend is in accord with the finding in molecular compounds that noble-metal halides with a halogen more electronegative than the metal itself experiences a relativistic destabilization of the bond, along with a bond length contraction [32]. For Ag we find a large bond stabilization of about 34.5\%. Bulk moduli and pressure derivatives change up to $7.5 \%$ (for $\mathrm{Ag}$ ) respectively up to $8.2 \%$ (for $\mathrm{AgCl}$ ) when the EOS of Murnaghan [27] is used. Corresponding fits of total HF energies versus the lattice parameters of $\mathrm{AgBr}$ and $\mathrm{Ag}$ are shown in Fig. 1 and Fig. 2., respectively.

Although scalar-relativistic HF calculations are not directly comparable with calculations using scalar-relativistic ionic pseudopotentials in the framework of the LDA [17] due to the missing treatment of correlation effects, our results suggest that relativistic effects in $4 \mathrm{~d}$ metals are overestimated when ionic pseudopotentials are used (for a comparison of absolute values see annotation in Table [II). This could explain why non-relativistic values 
of $4 \mathrm{~d}$ metals are slightly closer to experiment than scalar-relativistic ones (see Ref. [17] and references therein).

In Table $\mathbb{I \nabla}$ we compare crystal properties of $\mathrm{AgCl}$ at various levels of theory. All calculations are founded upon a periodic HF approach realized in the CRYSTAL program [19.33]. Results for different a posteriori density functional corrections and experimental values are included [29]. We find remarkable agreement between relativistic HF calculations using $H_{+}^{\mathrm{sf}_{1}}$ and relativistic ECP calculations using pseudopotentials of Hay and Wadt (see Ref. [29]) concerning the bulk modulus and the lattice parameter. Slight differences are observed for the molar formation energy and the pressure derivative. Corresponding fits of total HF energies per primitive unit cell versus the lattice parameter of $\mathrm{AgCl}$ are shown in Fig. 3 .

\section{CONCLUSIONS}

We have calculated scalar-relativistic effects of crystalline properties such as lattice parameters, molar formation energies, bulk moduli and pressure derivatives of various silver compounds with FCC structure by means of an implementation based on the Douglas-Kroll transformation within a periodic Hartree-Fock approach realized in the CRYSTAL program.

Good agreement between a relativistic effective-core potential calculation and the onecomponent Douglas-Kroll approach is obtained for most of the properties investigated for $\mathrm{AgCl}$. Though scalar-relativistic effects of silver compounds are expected to be small, remarkable changes of the Hartree-Fock binding energy and of the bulk modulus are observed.

\section{ACKNOWLEDGEMENTS}

The support of the "Deutsche Forschungsgemeinschaft" in the framework of the "Son-

derforschungsbereich 334: Wechselwirkungen in Molekülen" and of the European Science Foundation within the REHE program are gratefully acknowledged. 


\section{REFERENCES}

[1] C.L. Collins, K.G. Dyall, H.F. Schaefer III, J. Chem. Phys. 102 (1995) 2024.

[2] K. Molzberger, W.H.E. Schwarz, Theor. Chim. Acta 94 (1996) 213.

[3] M. Douglas, N.M. Kroll, Ann. Phys. 82 (1974) 89.

[4] B.A. Heß, Phys. Rev. A 33 (1986) 3742.

[5] R. Samzow, B.A. Heß, G. Jansen, J. Chem. Phys. 96 (1992) 1227.

[6] U. Kaldor, B.A. Heß, Chem. Phys. Lett. 230 (1994) 1.

[7] G. Jansen, B.A. Heß, Z. Phys. D. 13 (1989) 363.

[8] P. Schwerdtfeger, J.S. McFeaters, R.L. Stephens, M.J. Liddell, M. Dolg, B.A. Heß, Chem. Phys. Lett. 218 (1994) 362.

[9] B.A. Heß, C.M. Marian, S.D. Peyerimhoff, in: Modern electronic structure theory, Vol. 2, ed. D.R. Yarkony, (World Scientific, Singapore, 1995), p. 152.

[10] M.V. Ramana, A.K. Rajagopal, in: Advances in chemical physics, Vol. 54, eds. I. Prigogine, S.A. Rice, (Wiley, New York, 1983), p. 231.

[11] H. Gollisch, L. Fritsche, Phys. Stat. Sol. (b) 86 (1978) 145.

[12] H. Ebert, Phys. Rev. B 38 (1988) 9390.

[13] A.H. MacDonald, S.H. Vosko, J. Phys. C 12 (1979) 2977.

[14] W. Küchle, M. Dolg, H. Stoll, H. Preuss, J. Chem. Phys. 100 (1994) 7535.

[15] G.B. Bachelet, D.R. Hamann, M. Schlüter, Phys. Rev. B 26 (1982) 4199.

[16] N.E. Christensen, Int. J. Quantum Chem. 25 (1984) 233.

[17] C. Elsässer, N. Takeuchi, K.M. Ho, C.T. Chan, P. Braun, M. Fähnle, J. Phys.: Condens. Matter $2(1990) 4371$. 
[18] J. Ladik, J. Cizek, P.K. Mukherjee, in: Nato ASI Series B: Physics, Vol. 87, ed. G.L. Malli, (Plenum, New York, 1983), p. 305.

[19] R. Dovesi, C. Pisani, C. Roetti, M. Causà, V.R. Saunders, CRYSTAL88, (QCPE No. 577, Bloomington, 1989).

[20] C. Pisani, R. Dovesi, C. Roetti, Lecture notes in chemistry, Vol. 48 (Springer, Heidelberg, 1988).

[21] V.R. Saunders, C. Freyria-Fava, R. Dovesi, L. Salasco, C. Roetti, Mol. Phys. 77 (1992) 629.

[22] V.R. Saunders, in: Nato ASI Series C: Mathematical and Physical Sciences, Vol. 113, eds. G.H.F. Diercksen, S. Wilson (Reidel, Dordrecht, 1983) p. 1.

[23] R. Dovesi, Int. J. Quantum Chem. 29 (1986) 1755.

[24] C. Pisani, E. Aprà, M. Causà, Int. J. Quantum Chem. 28 (1990) 395.

[25] Handbook of Gaussian Basis Sets, in: Physical Sciences Data 24, eds. R. Poirier, R. Kari, I.G. Csizmadia (Elsevier, Amsterdam, 1985), Table 9.86.1, Table 17.33.1, Table 35.4.1.

[26] R.L. Martin, J. Phys. Chem. 87 (1983) 750.

[27] F.D. Murnaghan, Proc. Natl. Acad. Sci. (U.S.A.) 30 (1944) 244;

F. Birch, J. Geophys. Res. 91 (1986) 4949.

[28] P. Pyykkö, Chem. Rev. 88 (1988) 563.

[29] E. Aprà, E. Stefanovich, R. Dovesi, C. Roetti, Chem. Phys. Lett. 186 (1991) 329; 197 (1992) 338.

[30] C.P. Wood, N.C. Pyper, Chem. Phys. Lett. 81 (1981) 395.

[31] T. Ziegler, J.G. Snijders, E.J. Baerends, J. Chem. Phys. 74 (1981) 1271.

[32] P. Schwerdtfeger, M. Dolg, W.H.E. Schwarz, G.A. Bowmaker, P.D.W. Boyd, J. Chem. 
Phys. 91 (1989) 1762.

[33] R. Dovesi, V.R. Saunders, C. Roetti, CRYSTAL92, (Turin, Daresbury, 1992).

[34] K.F. Loje, D.E. Schuele, J. Phys. Chem. Solids 31 (1970) 2051.

[35] C.F. Bunge, J.A. Barrientos, A.V. Bunge, At. Data, Nucl. Data Tables 53 (1993) 113. 


\section{TABLES}

TABLE I. Basis sets used for relativistic ${ }^{\mathrm{a}}$ and non-relativistic atomic Hartree-Fock (HF) calculations for the ground state of $\mathrm{Ag}, \mathrm{F}, \mathrm{Cl}$ and $\mathrm{Br}$ and their total HF energies $\left(E^{\text {tot }}\right)$

\begin{tabular}{|c|c|c|c|c|c|c|}
\hline \multirow[t]{2}{*}{ Atom } & \multicolumn{3}{|c|}{ Outermost exponents ${ }^{\mathrm{b}}$} & \multirow[t]{2}{*}{ Method } & \multirow[t]{2}{*}{ State } & \multirow{2}{*}{$\begin{array}{l}E^{\mathrm{tot}} \\
\left(E_{\mathrm{h}}\right)\end{array}$} \\
\hline & $\mathrm{s}$ & $\mathrm{p}$ & $\mathrm{d}$ & & & \\
\hline \multirow[t]{5}{*}{$\mathrm{Ag}$} & \multicolumn{6}{|c|}{ Original basis: $(15 \mathrm{~s} 10 \mathrm{p} 8 \mathrm{~d})[26] \quad \longrightarrow$ Final basis: $(13 \mathrm{~s} 9 \mathrm{p} 8 \mathrm{~d})$} \\
\hline & \multicolumn{3}{|c|}{ Exponents deleted: $0.04(\mathrm{~s}) \quad 0.015(\mathrm{~s})$} & \multicolumn{3}{|l|}{$0.025(\mathrm{p})$} \\
\hline & & & & HF limit $[35]$ & ${ }^{2} \mathrm{~S}$ & -5197.6985 \\
\hline & $0.74(0.75)$ & 0.81 & $0.40(0.39)$ & nonrel. HF & ${ }^{2} \mathrm{~S}$ & -5197.0041 \\
\hline & 0.06 & 0.29 & $0.11(0.12)$ & rel. $\mathrm{HF}^{\mathrm{a}}$ & ${ }^{2} \mathrm{~S}$ & -5310.3549 \\
\hline \multirow[t]{4}{*}{$\mathrm{F}$} & \multicolumn{6}{|c|}{ Original basis: $(9 \mathrm{~s} 5 \mathrm{p})[25]$} \\
\hline & & & & HF limit $[35]$ & ${ }^{2} \mathrm{P}$ & -99.4094 \\
\hline & 1.21 & 0.94 & & nonrel. HF & ${ }^{2} \mathrm{P}$ & -99.3955 \\
\hline & 0.36 & 0.27 & & rel. $\mathrm{HF}^{\mathrm{a}}$ & ${ }^{2} \mathrm{P}$ & -99.4821 \\
\hline \multirow[t]{4}{*}{$\mathrm{Cl}$} & \multicolumn{6}{|c|}{ Original basis: $(11 \mathrm{~s} 7 \mathrm{p})[25]$} \\
\hline & & & & HF limit $[35]$ & ${ }^{2} \mathrm{P}$ & -459.4821 \\
\hline & 0.53 & 0.64 & & nonrel. HF & ${ }^{2} \mathrm{P}$ & -459.4382 \\
\hline & 0.19 & 0.18 & & rel. $\mathrm{HF}^{\mathrm{a}}$ & ${ }^{2} \mathrm{P}$ & -460.8422 \\
\hline \multirow[t]{4}{*}{$\mathrm{Br}$} & \multicolumn{6}{|c|}{ Original basis: (14s11p5d) [25] } \\
\hline & & & & HF limit $[35]$ & ${ }^{2} \mathrm{P}$ & -2572.4413 \\
\hline & 0.43 & 0.47 & 3.851 & nonrel. HF & ${ }^{2} \mathrm{P}$ & -2572.3577 \\
\hline & 0.16 & 0.14 & 1.32 & rel. $\mathrm{HF}^{\mathrm{a}}$ & ${ }^{2} \mathrm{P}$ & -2604.1851 \\
\hline
\end{tabular}

${ }^{\text {a }}$ Calculations using $H_{+}^{\mathrm{sf}_{1}}$.

b Only reoptimized exponents (au) are tabulated. Exponents which differ for the relativistic calculation are given in parentheses. 
TABLE II. Basis sets used for relativistic ${ }^{\mathrm{a}}$ and non-relativistic CRYSTAL88 Hartree-Fock (HF) calculations of the FCC crystals $\mathrm{Ag}, \mathrm{AgF}, \mathrm{AgCl}$ and $\mathrm{AgBr}$ and their total $\mathrm{HF}$ energies $\left(E^{\text {tot }}\right)$ with reference to the primitive unit cell

\begin{tabular}{|c|c|c|c|c|c|c|c|}
\hline \multirow{2}{*}{$\begin{array}{c}\text { Basis set } \\
\text { for }\end{array}$} & \multicolumn{3}{|c|}{ Outermost exponents ${ }^{b}$} & \multirow[t]{2}{*}{ FCC crystal } & \multirow[t]{2}{*}{ Method } & \multirow{2}{*}{$\begin{array}{c}\text { Accuracy } \\
\text { parameter }^{\mathrm{c}}\end{array}$} & \multirow{2}{*}{$\begin{array}{l}E^{\text {tot }} \\
\left(E_{\mathrm{h}}\right)\end{array}$} \\
\hline & $\mathrm{s}$ & $\mathrm{p}$ & $d$ & & & & \\
\hline $\mathrm{Ag}$ & \multicolumn{3}{|c|}{ Original basis: (15s10p8d) } & Final basis & $(13 \mathrm{~s} 9 \mathrm{p} 8 \mathrm{~d})[\mathrm{s}$ & e Table I] & \\
\hline \multirow[t]{2}{*}{$\mathrm{Ag}$} & 0.77 & 0.805 & 0.385 & $\mathrm{Ag}$ & nonrel. HF & 687713 & -5197.0191 \\
\hline & 0.10 & 0.22 & 0.13 & $\mathrm{Ag}$ & rel. $\mathrm{HF}^{\mathrm{a}}$ & 687713 & -5310.3751 \\
\hline
\end{tabular}

$\mathrm{Ag} \quad$ Original basis: (15s10p8d) $\longrightarrow$ Final basis: (13s9p8d) [see Table I]

F Original basis: (9s5p) [25]

\begin{tabular}{llll|lllll}
\hline $\mathrm{Ag}$ & 0.77 & 0.78 & 0.43 & & & & & \\
& 0.08 & 0.24 & 0.14 & $\mathrm{AgF}$ & nonrel. HF & 686613 & -5296.5548 \\
$\mathrm{~F}$ & 1.25 & 0.95 & & $\mathrm{AgF}$ & rel. HF & 686613 & -5409.9784 \\
& 0.38 & 0.28 & & & & & & \\
\hline
\end{tabular}

$\mathrm{Ag} \quad$ Original basis: (15s10p8d) $\longrightarrow$ Final basis: (13s9p8d) [see Table I]

$\mathrm{Cl} \quad$ Original basis: $(11 \mathrm{~s} 7 \mathrm{p})[25] \quad \longrightarrow$ Final basis: $(11 \mathrm{~s} 7 \mathrm{p} 1 \mathrm{~d})$

\begin{tabular}{|c|c|c|c|c|c|c|c|}
\hline \multirow[t]{2}{*}{$\mathrm{Ag}$} & 0.79 & 0.78 & 0.40 & & & & \\
\hline & 0.11 & 0.24 & 0.12 & $\mathrm{AgCl}$ & nonrel. HF & 686613 & -5656.6014 \\
\hline \multirow[t]{2}{*}{$\mathrm{Cl}$} & 0.54 & 0.70 & & $\mathrm{AgCl}$ & rel. $\mathrm{HF}^{\mathrm{a}}$ & 686613 & -5771.3441 \\
\hline & 0.20 & 0.20 & 0.14 & & & & \\
\hline $\mathrm{Ag}$ & \multicolumn{3}{|c|}{ Original basis: (15s10p8d) } & Final b & $(13 \mathrm{~s} 9 \mathrm{p} 8 \mathrm{~d})[\mathrm{s}$ & Table I] & \\
\hline $\mathrm{Br}$ & \multicolumn{7}{|c|}{ Original basis: (14s11p5d) [25] } \\
\hline \multirow[t]{2}{*}{$\mathrm{Ag}$} & 0.76 & 0.76 & 0.40 & & & & \\
\hline & 0.12 & 0.11 & 0.12 & $\mathrm{AgBr}$ & nonrel. HF & 687713 & -7769.5003 \\
\hline \multirow[t]{2}{*}{$\mathrm{Br}$} & 0.46 & 0.57 & 3.851 & $\mathrm{AgBr}$ & rel. $\mathrm{HF}^{\mathrm{a}}$ & 687713 & -7914.6672 \\
\hline & 0.16 & 0.19 & 1.30 & & & & \\
\hline
\end{tabular}


${ }^{\text {a }}$ Calculations using $H_{+}^{\mathrm{sf}_{1}}$.

b Only reoptimized exponents (au) are tabulated.

c The meaning of the accuracy parameters is described in Ref. [20] and are given in the order described in the user manual of Ref. [19]. All values are obtained using $29 \mathbf{k}$ points for the IBZ integration. 
TABLE III. Scalar-relativistic effects in the FCC crystals AgF, AgCl, AgBr and $\mathrm{Ag}^{\mathrm{a}}$

\begin{tabular}{llllll}
\hline \hline Property & Method & $\mathrm{AgF}$ & $\mathrm{AgCl}$ & $\mathrm{AgBr}$ & $\mathrm{Ag}^{\mathrm{b}}$ \\
\hline$a_{0}{ }^{\mathrm{c}, \mathrm{d}}(\mathrm{pm})$ & rel. HF & 517.5 & 593.7 & 620.7 & 445.6 \\
& nonrel. HF & 519.1 & 599.0 & 626.0 & 454.6 \\
& & & & 36.0048 & 13.3214 \\
$V_{0}\left(\mathrm{~cm}^{3} \mathrm{~mol}^{-1}\right)$ & rel. HF & 20.8664 & 31.5077 & 36.9350 & 14.1450 \\
& nonrel. HF & 21.0605 & 32.3591 & & -53.0 \\
$E_{0}\left(\mathrm{~kJ} \mathrm{~mol}{ }^{-1}\right)$ & rel. HF & -371.2 & -385.9 & -333.4 & -39.4 \\
& nonrel. HF & -407.5 & -417.7 & -362.8 & $298(295)$ \\
$B_{0}{ }^{\mathrm{d}}(\mathrm{kbar})$ & rel. HF & $459(459)$ & $306(309)$ & $273(272)$ & $322(323)$ \\
& nonrel. HF & $460(461)$ & $297(299)$ & $259(259)$ & $1.40(1.06)$ \\
$B_{0}^{\prime d}$ & & & & & \\
& rel. HF & $4.09(4.12)$ & $5.03(5.06)$ & $5.69(5.45)$ & $1.40(1.18)$ \\
\hline \hline
\end{tabular}


${ }^{\text {a }}$ Lattice parameter $a_{0}$, molar Volume $V_{0}$, molar Hartree-Fock $(\mathrm{HF})$ binding energy $E_{0}$ with reference to the HF ground-state energies of the free atoms and to the primitive unit cell, bulk modulus $B_{0}$ and pressure derivative $B_{0}^{\prime}$. The relativistic all-electron CRYSTAL88 HF calculation using $H_{+}^{\text {sf }_{1}}$ (rel. HF) is compared with a corresponding non-relativistic all-electron CRYSTAL88 HF calculation (nonrel. HF).

${ }^{b}$ Comparing non-relativistic and scalar-relativistic results of Ref. [17] scalar-relativistic effects lead to a bond length contraction (nearest-neighbor distance) of $5.7 \mathrm{pm}(1.9 \%)$, to a bond stabilization of $-31.8 \mathrm{~kJ} \mathrm{~mol}^{-1}(11.0 \%)$ and to an increase of the bulk modulus of $210 \mathrm{kbar}(19.8 \%)$ for $\mathrm{Ag}$. The molar formation energy in Ref. [17] is calculated with reference to the ground-state energy of the free silver atom and to the Wigner-Seitz cell volume which is identical to those of the primitive unit cell. The corresponding values for Ag in Table III are: bond length contraction $6.4 \mathrm{pm}(2.0 \%)$, bond stabilization $-13.6 \mathrm{~kJ} \mathrm{~mol}^{-1}(34.5 \%)$, decrease in the bulk modulus $24 \mathrm{kbar}(7.5 \%)$ (percentage with reference to non-relativistic values).

c Parameters of the restart option GEOM of the CRYSTAL88 program are for AgF (5.00), $\mathrm{AgCl}$ (5.90), $\mathrm{AgBr}(6.20)$ and $\mathrm{Ag}$ (4.45). The accuracy parameters are the same as in Table II.

d Ranges of bond distances used for fitting the EOS: AgF [490 - 540], AgCl [560 - 630], AgBr [605 - 670] and Ag [425 - 510] (all values in pm). The ranges have been chosen large enough to ensure numerical stability, and small enough to exclude inappropriately described regions of the potential curve at large bond distances, taking the inadequacy of the closed-shell HF approximation for near degenerate situations into account. Different EOSs [27] lead to same results for $a_{0}$ - otherwise values with an EOS of Birch [27] are given in parentheses. All mean square deviations of the fits are less than $0.6 \times 10^{-7}$. 
TABLE IV. Crystal properties of FCC $\mathrm{AgCl}$ at various levels of theory ${ }^{\mathrm{a}}$

\begin{tabular}{|c|c|c|c|c|c|c|}
\hline Method & $E^{\text {tot }}$ (a.u.) & $E_{0}\left(\mathrm{~kJ} \mathrm{~mol}^{-1}\right)$ & $a_{0}(\mathrm{pm})$ & $V_{0}\left(\mathrm{~cm}^{3} \mathrm{~mol}^{-1}\right)$ & $B_{0}(\mathrm{kbar})$ & $B_{0}^{\prime}$ \\
\hline nonrel. $H_{F}^{b, f}$ & -5656.6014 & -417.2 & 599.0 & 32.3591 & $297(299)$ & $4.65(4.75)$ \\
\hline rel. $\mathrm{HF}^{\mathrm{c}, \mathrm{f}}$ & -5771.3441 & -385.7 & 593.7 & 31.5077 & $306(309)$ & $5.03(5.06)$ \\
\hline rel. $\mathrm{ECP}^{\mathrm{d}, \mathrm{f}}$ & -159.7086 & -376.5 & 594.3 & 31.6000 & $307(312)$ & $5.38(5.44)$ \\
\hline rel. $\mathrm{ECP}^{\mathrm{e}}$ & -159.708 & -375.4 & 594.0 & 31.5532 & 330 & \\
\hline DFT1g & -160.656 & -554.0 & 570.0 & 27.8810 & 510 & \\
\hline $\mathrm{DFT}^{g}$ & -160.870 & -514.6 & 561.0 & 26.5811 & 560 & \\
\hline exp. ${ }^{h}$ & & -519.8 & 551.0 & $25.1848^{\mathrm{i}}$ & $535[417]$ & {$[7.00]$} \\
\hline
\end{tabular}


a Total energy $E^{\text {tot }}$ with reference to the primitive unit cell, molar formation energy $E_{0}$ with reference to the ground-state energies of the free atoms and to the primitive unit cell, lattice parameter $a_{0}$, molar volume $V_{0}$, isothermal bulk modulus $B_{0}$ and pressure derivative $B_{0}^{\prime}$.

b Non-relativistic all-electron Hartree-Fock (HF) calculation using the CRYSTAL88 program.

${ }^{\mathrm{c}}$ Relativistic all-electron Hartree-Fock (HF) calculation using the CRYSTAL88 program with $H_{+}^{\mathrm{sf}_{1}}$. ${ }^{\mathrm{d}}$ Relativistic effective-core potential (rel. ECP) calculation using the CRYSTAL92 program and ECPs for $\mathrm{Ag}$ and $\mathrm{Cl}$ given in Ref. [33].

e Relativistic effective-core potentials (rel. ECP) results calculated in Ref. [29] using nearly the same ECPs as in footnote d. (For Ag a $(5 \mathrm{~s} 5 \mathrm{p} 4 \mathrm{~d}) /[3 \mathrm{~s} 3 \mathrm{p} 1 \mathrm{~d}]$ GTF basis set is used instead of a (5s5p4d)/[3s3p2d] GTF basis set with $\xi=0.2108$ for $4 \mathrm{~d}$ instead of $\xi=0.2079$ for $4 \mathrm{~d})$.

f See footnote d of Table III.

g Correlation contribution calculated in Ref. [29] with a posteriori density functionals of Colle and Salvetti (DFT1) and Perdew (DFT2).

h Estimated experimental values (see Ref. [29] and references therein). The estimated values for $E_{0}, a_{0}$ and $B_{0}$ are an extrapolation of experimental results to $\mathrm{T}=0 \mathrm{~K}$ including a zero-point energy correction. All values are zero-pressure values. A zero-pressure value of the pressure derivative $B_{0}^{\prime}$ for the $\mathrm{B} 1$ phase of $\mathrm{AgCl}$ at $\mathrm{T}=0 \mathrm{~K}$ is not available. The estimated zero-pressure value $B_{0}$ at room temperature and the corresponding pressure derivative $B_{0}^{\prime}$ (see Ref. [34]) are given in parentheses. ${ }^{\mathrm{i}} V_{0}$ is deduced from $a_{0}$. 


\section{FIGURES}

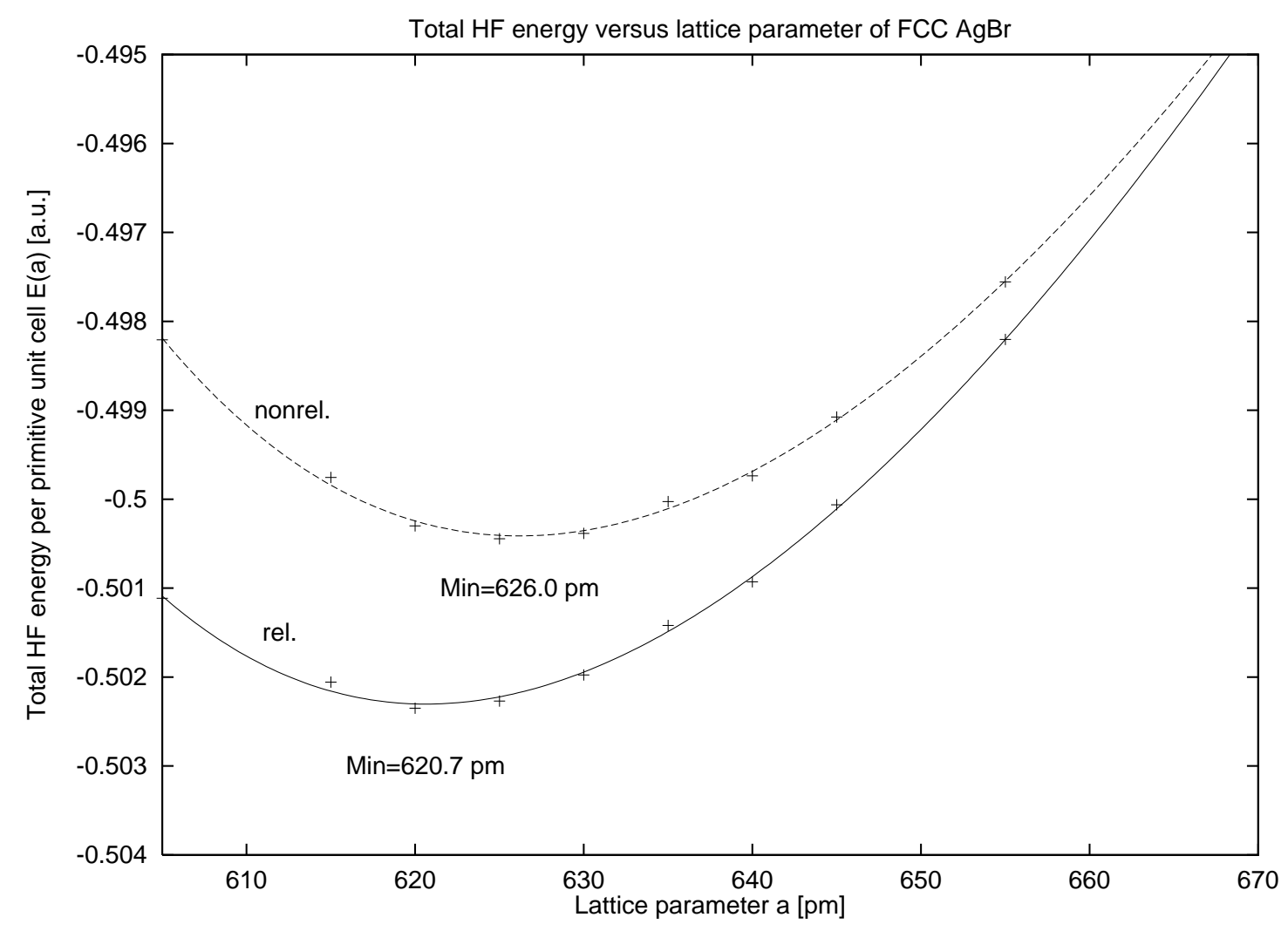

FIG. 1. Total Hartree-Fock (HF) energy versus lattice parameter of FCC AgBr. The fits using an EOS given by Murnaghan [27] are shifted by 7769.0 au (nonrel.) and by 7914.165 au (rel.). Abbreviations used: rel. (relativistic CRYSTAL88 HF calculation using $H_{+}^{\mathrm{sf}_{1}}$ ), nonrel. (non-relativistic CRYSTAL88 HF calculation). 


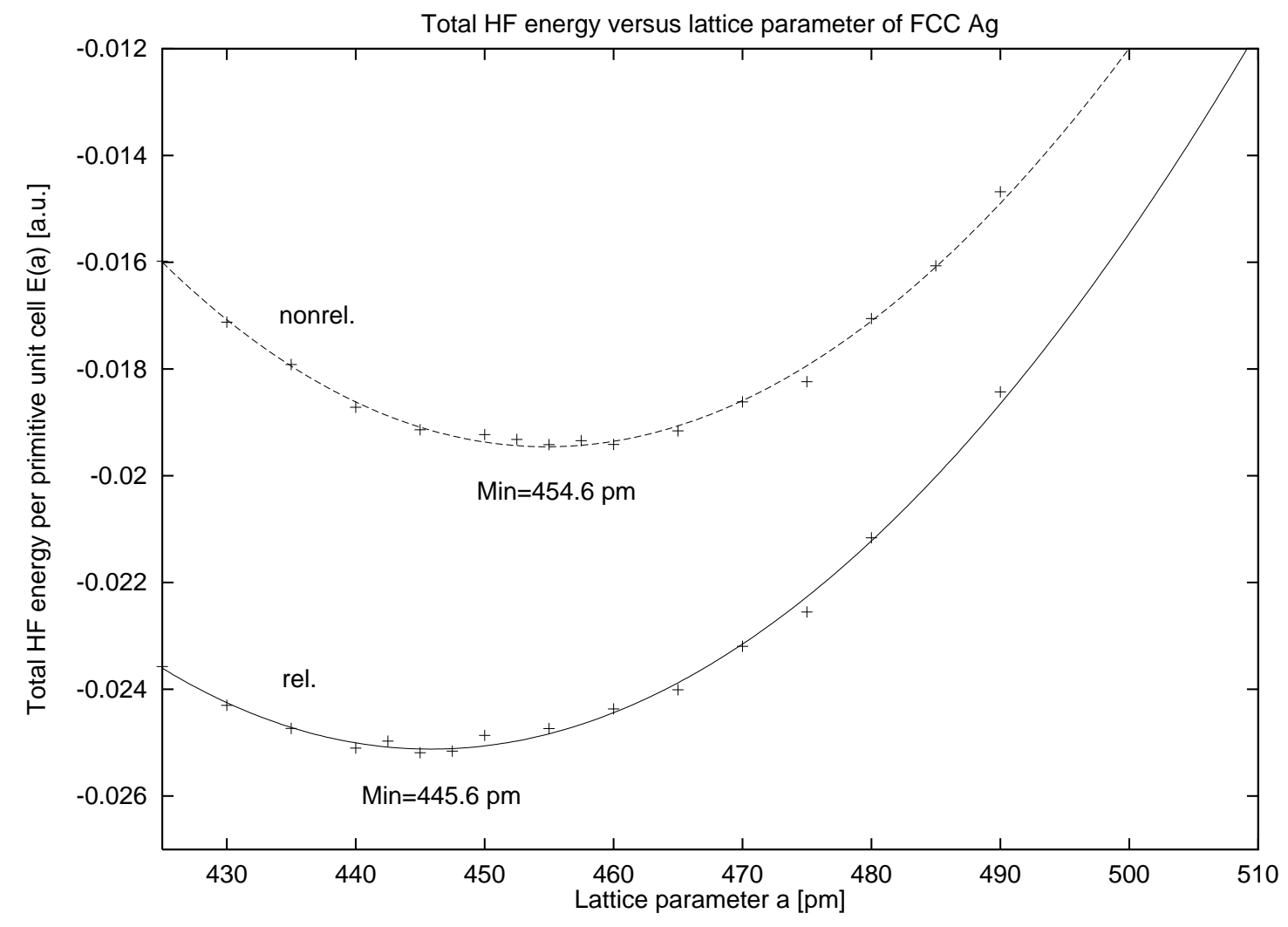

FIG. 2. Total Hartree-Fock (HF) energy versus lattice parameter of FCC Ag. The fits using an EOS given by Murnaghan [27] are shifted by 5197.0 au (nonrel.) and by 5310.35 au (rel.). Abbreviations used: rel. (relativistic CRYSTAL88 HF calculation using $H_{+}^{\mathrm{sf}_{1}}$ ), nonrel. (non-relativistic CRYSTAL88 HF calculation). 


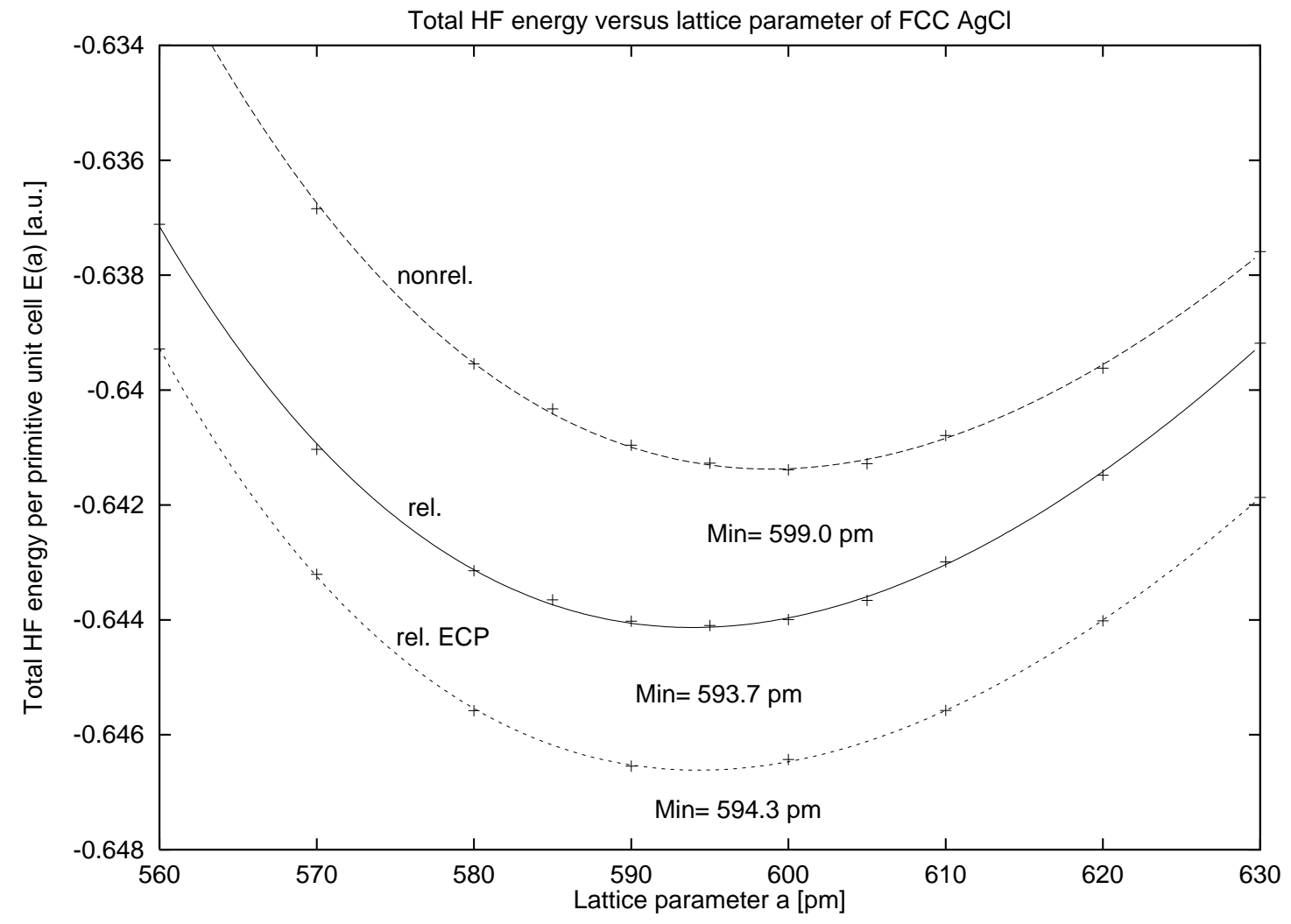

FIG. 3. Total Hartree-Fock (HF) energy versus lattice parameter of FCC AgCl. The fits using an EOS given by Murnaghan [27] are shifted by 5656.0 au (nonrel.), by 5770.7 au (rel.) and by 159.062 au (rel. ECP.). Abbreviations used: rel. (relativistic CRYSTAL88 HF calculation using $H_{+}^{\mathrm{sf}_{1}}$ ), nonrel. (non-relativistic CRYSTAL88 HF calculation), rel. ECP (relativistic effective-core potential CRYSTAL92 HF calculation [33]). 